\title{
La diacronia di anzi: considerazioni teoriche, dati e prime ipotesi ${ }^{1}$
}

\author{
Jacqueline VISCONTI \\ Università di Genova ${ }^{1}$ \\ j.visconti@unige.it
}

Recibido: 10/06/2015

Aceptado: 28/10/2015

\begin{abstract}
RIASSUNTO
Nel contributo si presentano i primi risultati di uno studio sulla diacronia della particella italiana anzi. Nei dati del XIII secolo, tratti dall'Opera del Vocabolario dell'Italiano (OVI), coesistono per anzi valore temporale (di precedenza) e valore "contrastivo-correttivo" (Bazzanella 2003). Quest'ultimo si esplica in particolare nel costrutto 'non p, anzi q', in cui la negazione porta tipicamente su un'entità già presente nel discorso, un punto di vista di altri, che viene refutato e sostituito con q; anzi può essere parafrasato con congiunzioni avversative quali ma o bensi o con al contrario. Nei dati del XX secolo, tratti dalla Letteratura Italiana Zanichelli (LIZ), permane solo l'uso contrastivo-correttivo; oltre al costrutto precedente si nota un uso privo di negazione su $\mathrm{p}$, in cui q è presentata come una formulazione "migliore", o un'aggiunta ad ulteriore specificazione: 'p, anzi q'. I dati del web corpus Risorse Dinamiche dell'Italiano (RIDIRE), del Lessico dell'Italiano Parlato (LIP) e dell'Integrated Reference Corpora for Spoken Romance Languages (C-ORAL-ROM) evidenziano inoltre impieghi con portata sull'illocuzione o come segnale enfatico di inversione della polarità: 'non $\mathrm{p}$, anzi!'.

$\mathrm{Da}$ questa prima ricognizione, alcune ipotesi appaiono plausibili e meritevoli di approfondimento: (i) il ruolo della struttura comparativa 'anzi p che q' nel passaggio dal valore temporale al valore di preferenza e successivamente di correzione di anzi; (ii) l'apparente diffusione, a partire dal XV secolo, dell'uso correttivo senza inversione di polarità; (iii) l'aumento di portata della particella ed il suo spostamento verso la periferia dell'enunciato.
\end{abstract}

Parole chiave: anzi, congiunzioni avversative, correzione, mutamento semantico.

${ }^{1}$ Dipartimento di Italianistica, Romanistica, Antichistica, Arti e Spettacolo, Via Balbi 6, I-16126 - Genova.

Ringrazio Carla Bazzanella, Nicola De Blasi, Davide Ricca, Elizabeth Traugott e due referees anonimi per i loro ottimi suggerimenti, Margarita Borreguero per questa ed altre belle occasioni di scambio. 


\title{
The diachrony of anzi: \\ Theoretical framework, data and preliminary results
}

\begin{abstract}
This paper presents the preliminary results of a diachronic investigation of the Italian particle anzi. In the XIII century data, from the Opera del Vocabolario dell'Italiano (OVI), temporal (precedence) and "contrastive-corrective" values coexist (Bazzanella 2003). The latter, in particular, is realized by the construction 'non p, anzi q', in which the negation is typically on an entity that is already in the discourse; another point of view, which is denied and replaced by q; anzi may be paraphrased by adversative conjunctions such as ma 'but' or bensi 'but (rather)' or by al contrario 'on the contrary'. In the XX century data, from the Letteratura Italiana Zanichelli (LIZ), only the contrastive-corrective use is found; as well as in the previous construction, anzi is used without negation on $\mathrm{p}$, while $\mathrm{q}$ is presented as a "better" formulation, or as a further specification: 'p, anzi q'. Moreover, data from the web corpus Risorse Dinamiche dell'Italiano (RIDIRE), the Lessico dell'Italiano Parlato (LIP) and the Integrated Reference Corpora for Spoken Romance Languages (C-ORAL-ROM) highlight that anzi can be used with scope on the illocution or as an emphatic marker of polarity inversion: 'non p, anzi!'.

On the basis of these preliminary observations, the following hypotheses are put forth for further investigation: (i) the role of the comparative structure 'anzi p than q' in the shift from temporal to preference and subsequently to correction value; (ii) the apparent spread, from the XV century onwards, of the corrective use without polarity inversion; (iii) the particle scope increase and its movement towards the periphery of the utterance.
\end{abstract}

Key words: anzi, adversative conjunctions, correction, semantic change.

SOMMARIO: 1 . Introduzione 2. Le origini 3 . Verso la periferia 4. Prime ipotesi e linee di ricerca.

\section{INTRODUZIONE}

Per un approccio che miri a identificare un minimo comune denominatore tra le relazioni di tipo avversativo, la correzione è quella più sfuggente. Come nota già Foolen (1991: 87): «Several authors have capitulated on this point, suggesting that correction is really something different than either contrast or denial of expectation [...]. Many languages, moreover, take this fact into account as they employ a form for correction that differs from the standard adversative conjunction». Non solo, dunque, la correzione si presenta come qualcosa di molto diverso dal contrasto o dalla controaspettatività tipicamente associati ai connettivi avversativi, ma diverse lingue presentano per tale relazione una congiunzione diversa e dedicata. Queste lingue, quali, ad esempio, lo spagnolo, il tedesco, lo svedese, l'ebraico, sono dette lingue Aber/Sondern da Lang (1984: 252). 
In questo contributo ci occuperemo di anzi, una particella ${ }^{2}$ per cui la correzione è, in italiano contemporaneo, una funzione centrale. L'indagine è ai suoi inizi; nelle pagine che seguono proporremo alcune ipotesi sulle origini della particella (1), tracceremo un possibile cammino diacronico (2) e delineeremo un percorso di ricerca (3).

I dati provengono per l'italiano antico dal corpus dell'Opera del Vocabolario dell'Italiano (OVI), per l'italiano contemporaneo scritto dalla Letteratura Italiana Zanichelli (LIZ) e dal web corpus Risorse Dinamiche dell'Italiano (RIDIRE), per il parlato dal Lessico dell'Italiano Parlato (LIP) e dall'Integrated Reference Corpora for Spoken Romance Languages (C-ORAL-ROM).

\section{LE ORIGINI}

Bazzanella (2003), a mia conoscenza, l'unico lavoro finora dedicato ad anzi, ne riconduce lo sviluppo al latino ANTE 'davanti, prima'. Come mostra la sua analisi, mentre ANTE aveva in latino funzione spaziale, temporale e comparativa, anzi presenta in italiano antico usi temporali e "contrastivo-correttivi", unica funzione, quest'ultima, a sopravvivere nell'italiano contemporaneo. Il processo è considerato da Bazzanella un caso di "deriva modale", che da valori spaziali e temporali procede ai valori di comparazione, poi contrasto. Lo schema proposto, da non intendersi in senso strettamente unidirezionale, è il seguente (Bazzanella 2003: 135):

CORRELAZIONE — OPPOSIZIONE — CONFRONTO — PREFERENZA — CONTRASTO CORREZIONE

Partendo da questa analisi, il nostro lavoro prenderà due direzioni: la prima cercherà di precisare la relazione indotta da anzi attraverso i secoli, e, in particolare, di identificare i contesti "critici", correttivo. La seconda direzione si concentrerà sul correlato strutturale, rimasto finora inesplorato, di questo mutamento semantico.

Il quadro teorico in cui si inscrive l'indagine è quello delineato in Visconti (2015), dove l'idea centrale è che il mutamento linguistico non interessi entità in isolamento, bensì sia indotto dalla contiguità con componenti co-testuali e contestuali, e che la frequente associazione di un lessema, o di un costrutto, con

${ }^{2}$ L'uso del termine "particella" intende ovviare alla difficoltà di una caratterizzazione sintattica univoca per gli usi di anzi in italiano contemporaneo.

${ }^{3}$ Critical contexts sono per Diewald (2002) quei contesti, opachi sia semanticamente sia strutturalmente, che innescano il processo di rianalisi (si veda anche Diewald / Smirnova 2012), analoghi ai bridging contexts di Heine (2002). 
determinati contesti, appunto critici (o ponte), lo porterebbe ad assorbire le proprietà associate a tali contesti.

Per capire come l'operazione cognitiva e comunicativa realizzata da anzi evolva nei secoli e per cercare di delimitare più chiaramente ciò che è indotto da anzi e ciò che è invece un effetto contestuale, partiremo dal confronto di 60 esempi del XIII secolo, tratti dall' ovI, con altrettanti del XX secolo, tratti dalla LIZ.

Per cominciare, guarderemo agli impieghi monologici del costrutto 'p, anzi q'.

I parametri su cui ci concentreremo riguardano 3 aspetti:

(i) la presenza $v s$ assenza di una negazione esplicita su p, ad esempio ' $\underline{\text { non era }}$ felice, anzi era triste' $v s$ 'stava bene, anzi molto bene';

(ii) la portata di anzi, se cioè la relazione indotta porti su un costituente, anche frasale, oppure su porzioni testuali più ampie;

(iii) la natura della relazione tra $\mathrm{i}$ due congiunti $\mathrm{p}$ e q; ad esempio, nel caso di predicati, se siano antonimi, iperonimi, ecc.

2.1. Nell' ovi, i primi 36 esempi hanno tutti valore temporale; in un primo impiego anzi è seguito da un oggetto diretto (1):

(1) Pagava(mo) $\mathrm{p}(\mathrm{er})$ Baldovino suo fratello, tre di $a(n) z i \mathrm{k}$. Agosto (Doc. fior, 1211, $33.26[\mathrm{OVI}])$.

In un secondo costrutto, sempre con valore temporale, anzi è seguito da un complemento frasale introdotto da che:

(2) Pregò Domenedio e disse: Segniore Dio io ti prego, che tu mi facci due cose anzi ch'io muoia (Andrea da Grosseto, 1268 (tosc.) L. 3, cap. 2, 182.5 [OVI]).

Nei 24 esempi successivi, anzi ha valore "contrastivo-correttivo", nella terminologia di Bazzanella (2003); è cioè usato per introdurre quello che il locutore considera il predicato più appropriato per un dato soggetto, dopo aver negato il primo predicato (' $\mathrm{X}$ non è $\mathrm{p}$, anzi è q'):

(3) le leggi del tiranno non sono leggi, anzi sono corruzione del paese o del reame (Egidio Romano volg., 1288 (sen.) L. 3, pt. 2, cap. 23, 267.28 [OVI]).

(4) ché quelli che non teme Dio non è forte, anzi è pazzo (Egidio Romano volg., 1288 (sen.) L. 1 pt., 2 cap. 13, 43.16 [OVI]).

Di questi esempi, tutti contengono una negazione esplicita su p, come (3) e (4) sopra; solo due non la contengono, come (5):

(5) sofferente seraggio al so piacere,/ di bon[o] core e di pura lëanza / la servo umilemente, anzi vorria per ella pena avere (Giacomo da Lentini, 1230/50 (tosc.) 15, v. 3, 191.13 [OVI]). 
La portata di anzi è nei 60 casi esaminati un predicato, o un costituente frasale, come negli esempi precedenti, o ambigua tra i due casi. Il primo, isolato, esempio, in cui anzi ha una portata lievemente più ampia è (6):

(6) se' ingannato se tu credi, che la ventura sempre ti dea prosperità e ti nutrichi; perciò che fino a ora ti abbia nutrito. Anzi puo' credere tutto '1 contrario (Andrea da Grosseto, Trattati morali di Albertano da Brescia volgarizzati (ed. Selmi), 1268 (tosc.) L. 2 cap. 41, 136.18 [OVI]).

Negli esempi contrastivo-correttivi del XIII secolo, i due predicati paralleli legati da anzi sono, con poche eccezioni, antonimici: essere renduto $v s$ spendere; stinguersi $v s$ allumarsi; ralegrare $v s$ umiliare; guarire $v s$ morire; dritto $v s$ torto; conoscersi $v s$ uccidere l'un l'altro; laudare $v s$ vituperare; da guidar donare $v s$ degno di pena; materia di rettorica $v s$ materia di poete; fuor $v s$ dentro; fretoloso, furioso $v s$ lento; imparare da rio maestro $v s$ schifare secondo veleno; passare dì ozioso vs divider notte con studio; amici vs nemici da odiare:

(7) Non guarisca, anzi sia morto; non sia fatto dritto, anzi torto! (Ruggieri Apugliese (ed. Contini), XIII sec. (sen.) 3 v. 84906.14 [OVI]).

(8) E dice "crudeltade" perciò che 'l padre e'l figliuolo non si conosceano, anzi uccidea l'uno l'altro (Brunetto Latini, Rettorica, 1260-61 (fior.), 23.21 [OVI]).

Una costruzione interessante è quella in cui anzi che è interrotto da un sintagma, come in 'anzi $\mathrm{p}$ che q', che prende quindi la forma di una struttura comparativa esempi (9) e (10); tale struttura, a mio avviso, potrebbe aver giocato un ruolo importante nell'aprire la via al passaggio dal valore temporale a quello correttivo:

(9) Affaticati anzi per te che per altrui... (Fiori di filosafi, 1271-75 (fior.), 119.9 [TLIO].

(10) Li buoni debbono anzi amare lo giudice che temere (Andrea da Grosseto (ediz. Selmi), 1268 (tosc.) L. 2 cap. 40, 133.26 [TLIO]).

Porre due stati di cose in una relazione di sequenzialità temporale, in contesti deontici o di referenza futura, può infatti suggerire un'inferenza di precedenza e priorità, come ben documentato in studi anche su lingue diverse (oltre a Bazzanella (2003) su ANTE, si veda Cuenca (1992: 187) sul catalano ans, Rodriguez Somolinos (2002) sul francese antico ainz, Traugott e König (1991) e Traugott (i.c.s.) sull'inglese antico hraðor > rather).

Il passaggio successivo da precedenza / priorità a contrasto / sostituzione / correzione è a mio vedere favorito da contesti, come (9) e (10) qui sopra, in cui la comparazione concerne due stati di cose che non solo possono essere posti su una scala, ma che possono inoltre essere concettualizzati come mutualmente esclusivi 
('te $v s$ gli altri'; 'amare $v s$ temere'), come negli esempi latini del tipo MORTUUS ANTE QUAM SERVUS.

Come notano Traugott / König (1991: 206) per hraðor, il comparativo di hrcepe 'quick, early', che in inglese antico aveva valore sia di precedenza temporale sia di preferenza, in contesti del tipo 'his daughter, who had chosen the Lord, would rather die than get married' ('sua figlia, che aveva scelto il Signore, sarebbe piuttosto morta che prendere marito'), si ha un'inferenza di «rejection of one alternative», come in 'die so that she would not have to get married' ('morire per non prendere marito'). Tale inferenza di rifiuto dell'alternativa in q nel costrutto 'rather $p$ than $q$ ' apre al passaggio da preferenza a correzione: 'prima/meglio $\mathrm{p}$ che $q$ '> $p$, non q'.

Il costrutto 'anzi $p$ che q' crea dunque i presupposti per il passaggio alla configurazione 'non p, anzi q': in essa il senso di priorità / precedenza indotto dal lessema, la refutazione di $\mathrm{p}$ e l'asserzione di un predicato concettualizzabile come antitetico si combinano a creare il valore sostitutivo / correttivo.

2.2. Compiamo ora un salto temporale agli esempi del XX secolo; useremo i dati della LIZ e, secondariamente, quelli del corpus RIDIRE, in quanto testi scritti, idealmente più vicini, con tutti i caveat del caso, agli esempi dell'OVI, almeno rispetto ai dati del parlato contemporaneo contenuti nel LIP e nel C-ORAL-ROM ${ }^{4}$.

Nella LIZ, dei 60 esempi del XX secolo solo 30 sono di tipo monologico; confrontando li con i 24 di tipo "contrastivo-correttivo" del XIII secolo, per quanto l'analisi di così pochi dati abbia solo valore indicativo, essi appaiono subito diversi: solo 12 manifestano una negazione; di questi, 6 ricalcano la struttura 'non p, anzi q' tipica del XIII secolo, mentre negli altri casi la negazione è espressa da senza e la refutazione molto indiretta, come in:

(11) L'aveva invidiata allora, sentendosi inferiore a lei per giovinezza e bellezza, ma senza sdegno e senz'odio perché allora stimava che non era colpa di colei se il marchese l'aveva voluta e se l'era tenuta in casa. Ne aveva avuto anzi compassione, povera giovane! (L. Capuana, Il Marchese di Roccaverdina, cap 31.53 [LIZ]).

(12) S'accaloravano così man mano ciascuno a dire il fatto suo e a difendere il suo interesse, senza pensare più né a sposa né a sposalizio. Anzi don Nunzio alzava la voce per far notare che al giorno d'oggi sulla dote che doveva garentire la zolfara non si poteva più contare... (G. Verga, Dal tuo al mio (romanzo), XX sec., cap. 1. 419 [LIZ]).

\footnotetext{
${ }^{4}$ Un primo inevitabile approfondimento dovrà affrontare la spinosa questione di generi e tipi testuali, soprattutto in relazione alla dimensione diamesica, per evitare che differenze nell'uso della particella siano invece dovute a fattori, appunto, diamesici o diafasici.
} 
18 casi, quindi quasi due terzi degli esempi a campione, non presentano negazione su p:

(13) Era tutto sudato e gongolante anche lui, il bravo don Rocco, buon parente e buon amico - anzi più che parente e amico, in quell'occasione (G. Verga, Dal tuo al mio (romanzo), XX sec., cap. 1. 150 [LIZ]).

(14) Io solo, Lazzaro Chieco, violoncellista di camera, anzi di anticamera del Padre Eterno, lo sono! (A. Fogazzaro, Piccolo mondo moderno, XX sec., cap. 3,4.5 [LIZ]).

Si nota cioè, anche da questo primo spoglio, la diffusione del costrutto privo di negazione su $\mathrm{p}$, esemplificato in (5), che nel XIII secolo era in netta minoranza ${ }^{5}$. Nell'uso prevalente in italiano antico $\mathrm{p}$ viene negato e sostituito con q; anzi può essere parafrasato con congiunzioni avversative quali 'ma' o 'bensì' o con 'al contrario'; la negazione porta tipicamente su un'entità già presente nel discorso, un punto di vista di altri, che viene refutato - che il punto di vista negato sia già in qualche modo presente nell'universo discorsivo è confermato dalla frequente associazione di anzi a mica, una forma di negazione che in italiano antico (Visconti 2007), come in italiano contemporaneo (Cinque 1976), riprende entità date, attive nel co-testo precedente:

(15) E venimo un sabato sera al mio abitacolo, ma 'libretto no lascia' io mica, anzi il ne recai, ché molto desiderava io la compagnia de le sante parole che v'erano entro (Storia San Gradale, XIV (fior.) 26.33 [TLIO]).

(16) Eu ricuntiròmoduquilli li quali, avendu alcuni hominisuspetti, appirumultu grandi et exquisita guardia di si medemmi. Nìnincuminzirò mica da luplù mestino, anti incuminzirò da quillu, lu quali intra poki era tinutu la plùbeatu (Accurso di Cremona, 1321/37 (mess.) 9.14 [TLIO]).

Nell'altro costrutto si ha invece l'introduzione di $\mathrm{p}$ da parte dello stesso locutore, che non lo nega,ma lo sostituisce con q, una formulazione "migliore", o un'aggiunta ad ulteriore specificazione, come nell'esempio (17) in cui anzi è introdotto dalla congiunzione copulativa $e$; la parafrasi con 'ma', 'bensì' o 'al contrario' non è più possibile ${ }^{6}$ :

(17) Però degli ufficiali malcontenti ve ne dovevano essere; e anzi, alcuni dicevano che quelli del Fieramosca [...] erano stati visti con le lagrime agli occhi e il dolore sul viso (G. C. Abba, Storia dei Mille. L'agitazione per la Sicilia, XX sec., 12 [LIZ]).

${ }^{5}$ Da un primo riscontro dei dati della LIZ questo costrutto resta minoritario fino al XIV secolo e si diffonde poi rapidamente a partire dal XV secolo.

"I due impieghi sono detti "avversativo" («sempre dopo una frase di senso negativo») e "correttivo-accrescitivo" nel DISC. 
La correzione / precisazione può concernere aspetti diversi, dalla cardinalità (più mele vs una: 18), (una mano vs due: 19), alla modalità epistemica (parere $v s$ essere certamente: 20); (dover essere vs essere certo: 21$)$ :

(18) $[\ldots]$ gli erano rimaste poche foglie sbiadite; e nelle punte dei suoi fuscelli i segni dove stavano le mele: una sola, anzi, gialla e grinzosa (F.Tozzi, Bestie, XX sec., 52.7 [LIZ]).

(19) /LAuri/A voi la mano. Anzi, tutte e due le mani (G. Giacosa, Come le foglie, At. 1, sc. 11, XX [LIZ]).

(20) Una volta la minestra mi parve sciocca; anzi, era certamente (F. Tozzi, Bestie, XX sec., 45.1 [LIZ]).

(21) Sì! Il nemico doveva essere, anzi era certo non lontano, già in posizione(G. C. Abba, Storia dei Mille. Il nemico, XX sec.,7 [LIz]).

Dato comune a questo tipo di esempi è la presenza di una componente di gradazione, di scalarità, tra i due predicati (22) o tra i due stati di cose (23):

(22) No, no; sto bene, molto bene anzi. Che mi manca? (L. Capuana, Il Marchese di Roccaverdina, XX sec.,cap 25.29 [LIZ]).

(23) M'era rimasto tanto da andare a pranzo, e alle due fui puntualissimo dal notaio. Anzi arrivai prima degli altri (F. Tozzi, Giovani, La casa venduta, sec. XX, 119 [LIZ]).

Una possibile spiegazione per questo sviluppo, in linea con il quadro teorico prescelto, è che mentre in italiano antico è l'intera costruzione a indurre il senso sostitutivo / correttivo - 「reputazione + precedenza / priorità di un predicato antitetico] - , in italiano contemporaneo la particella ha ormai assorbito e convenzionalizzato il valore sostitutivo-correttivo, tanto da essere in grado di esprimerlo in altri contesti, non refutativi, e, come vedremo, con portata su porzioni testuali più ampie e sull'illocuzione.

L'uso "antico", con la negazione su p, permane, anzi si routinizza al punto da non richiedere l'esplicitazione di q; si hanno così quegli usi di anzi "sospeso", ravvisabili per ora soprattutto nel parlato, su cui torneremo nella sezione successiva:

(24) LEO: <perché io > mi ricordo che / che / da Davoli / che fra l'altro non è neanche tra i più economici che esistano a i’ mondo/ anzi...

GPA: [.] $\mathrm{mh} / /$

LEO: entrò una ragazza /voleva comprare (C-ORAL-ROM) 
(25) GIA: ma se si facesse la settimana di Pasqua / invece? o di lunedì o martedì // no? RST: prima di Pasqua?

GIA: eh icché c'è a Pasqua? 'un c'è mica nulla // anzi //

LAU: $\mathrm{mh} / /$ poi è una domenica (C-ORAL-ROM)

La sospensione non avviene negli usi del secondo tipo, dove q non è così facilmente calcolabile a partire da non p come negli esempi del primo tipo:

(26) La situazione del calcio italiano, anzi dei calciatori italiani (RIDIRE)

*La situazione del calcio italiano, anzi...

(27) Wittgenstein istituisce un confronto e stabilisce, anzi, un'analogia (RIDIRE)

*Wittgenstein istituisce un confronto e stabilisce, anzi...

In sintesi,anche se $\mathrm{i}$ dati dei secoli intermedi saranno necessari a confermare l'ipotesi, i fenomeni riscontrati si riconducono alla convenzionalizzazione in anzi del valore sostitutivo-correttivo e all'evoluzione della relazione verso una correzione più "metalinguistica" che contenutistica, in cui non si contrastano cioè stati di cose o predicati opposti, di cui uno negato, ma si propone una formulazione più appropriata a sostituzione del primo predicato. Per usare la distinzione introdotta da Manzotti (1999) a proposito di cioè tra «correzione totale» e «parziale», la relazione espressa da anzi appare evolvere dal primo al secondo tipo: nel primo caso «prima di sostituirgliene una più adeguata, il locutore respinge in toto (o fa come se così fosse) l'espressione che giudica inadeguata» (Manzotti 1999: 199), mentre nel secondo tipo «mostra (finge) di accogliere almeno in parte l'espressione $\mathrm{A}$ prima di sostituirle una $\mathrm{B}$ parzialmente diversa, e parzialmente più adeguata» (Manzotti 1999: 199).

Guardiamo ora al correlato strutturale di questi sviluppi.

\section{VERSO LA PERIFERIA}

In italiano antico anzi manifesta un grado di integrazione sintattica elevato, più alto negli usi temporali - esempi (1) e (2) sopra, ma comunque alto negli usi contrastivo-correttivi - da (3) a (8).

Negli esempi del XX secolo la portata appare invece più ampia, sia nello scritto letterario:

(28) Anche qui avete da fare con galantuomini, don Nunzio - riprese il notaio mettendogli una mano sulla spalla. - $\mathrm{Ci}$ accomoderemo senza bisogno dell'usciere. - Anzi si voltò a quest'ultimo, che stava preparando i ferri del mestiere: - Per ora non abbiamo bisogno di voi, don Calogero (G. Verga, Dal tuo al mio (romanzo), XX sec., cap. 2, 199 [LIZ]). 
sia, ancor di più, negli esempi di parlato, in cui anzi porta tipicamente su atti linguistici, come in (29) e (30):

(29) ma non ci dice tutto \# l'etimologia della parola perché \# se io ti dico eh filologia eh tu sai cosa significa perché ne abbiamo parlato anzi cos'è la filologia * (LIP)

(30) sì sì come no * vieni domani anzi sai che pensavo * che per la casa di andare giovedì * eh venerdì pomeriggio presto \# così veniva pure XYZ (LIP)

Una seconda innovazione è che nell'italiano contemporaneo anzi è spesso alla periferia dell'enunciato, sia alla sinistra sia alla destra, come negli usi che avevamo definito come "sospesi" nella sezione precedente, in cui esso appare come un segnale enfatico dell'inversione di polarità:

(31) anche perché voi state in difetto ha capito * non è una questione di \# che uno non ve vuole aiuta' anzi [LIP]

(32) sì si può dire anche perché s'è avuto sempre dei buoni rapporti con eh specialmente i tifosi viola anzi $*$ [LIP]

Questa evoluzione da posizioni interne al nucleo a posizioni periferiche porta con sé una serie di domande: (i) Che cosa motiva l'aumento di portata? (ii) Che funzione hanno gli usi periferici? (iii) Anzi sta diventando una "particella finale" (final particle) nel senso di Hancil et al.(2015: 4): «elements that have little or no lexical or conceptual, but predominantly procedural meaning $[\ldots]$ in that they provide an interpretive cue to the hearer asto how understand the sentence or utterance they accompany»?

\section{PRIME IPOTESI E LINEE DI RICERCA}

Da questa prima ricognizione dei dati, alcune ipotesi appaiono plausibili e meritevoli di approfondimento in diacronia, con estensione dell'indagine ai contesti dialogici e con particolare attenzione alla diamesia:

1. Il ruolo della struttura comparativa nel passaggio dal valore temporale al valore di preferenza e successivamente di correzione di anzi, ipotesi da valutarsi nel contesto della derivazione di congiunzioni avversative quali ma o il catalano mes dal latino MAGIS tramite una struttura comparativa (si veda, rispettivamente, Marconi e Bertinetto 1984 e Cuenca 1992) ${ }^{7}$.

\footnotetext{
${ }^{7}$ In questo contesto sarà interessante un raffronto con la diacronia di piuttosto (v. Mauri / Giacalone Ramat, in questo volume).
} 
2. La relazione tra $\mathrm{i}$ due impieghi individuati per anzi, e in particolare l'apparente diffusione, a partire dal XV secolo, dell'uso correttivo senza inversione di polarità.

3. L'aumento di portata della particella su porzioni testuali più ampie, ad atti linguistici nel parlato, e il suo spostamento verso la periferia dell'enunciato.

Se l'uso della particella in italiano antico è ancora vicino a quello di congiunzioni avversative come $m a$ o bensì, questi ultimi aspetti della sua evoluzione lo differenziano radicalmente nei secoli successivi. Oltre allo studio diacronico, il vaglio di ampi corpora di parlato consentirà di valutare l'espansione di anzi a impieghi con portata sull'illocuzione o come segnale enfatico di inversione della polarità.

\section{RIFERIMENTI BIBLIOGRAFICI}

BAZZANELlA, Carla (2003): «Dal latino ANTE all'italiano anzi: la 'deriva modale'», in Alessandro Garcea (a cura di), Colloquia Absentium. Studi sulla comunicazione epistolare in Cicerone, Torino, Rosenberg, pp. 123-140.

CINQUE, Guglielmo (1991 [1976]): «Mica: note di sintassi e pragmatica», Teoria linguistica e sintassi italiana, Bologna, Il Mulino, pp. 311-323.

CUENCA, Maria Josep (1992): «Sobre l'evoluciò dels nexes conjunctus en català», Llengua y Literatura 5, pp. 171-213.

DIEWALD, Gabriele / SMIRNOVA, Elena (2012): «Paradigmatic integration: The fourth stage in an expanded grammaticalization scenario», in Kristin Davidse, Tine Breban, Lieselotte Brems, Tanja Mortelmans (eds.), Grammaticalization and Language Change: New reflections, Amsterdam/Philadelphia, John Benjamins, pp. 111-134.

FoOLEN, Ad (1991): «Polyfunctionality and the semantics of adversative conjunctions», Multilingua 10 (1-2), pp. 79-92.

HANCIL, Sylvie / HASElowe Alexander / Post, Margje (eds.) (2015): Final Particles, Berlin/Boston, De Gruyter Mouton.

LANG, Ewald (1984): The Semantics of Coordination, Amsterdam/Philadelphia, John Benjamins.

MANZOTTI, Emilio (1999): «Spiegazione, riformulazione, correzione, alternativa: sulla semantica di alcuni tipi e segnali di parafrasi», in Lucia Lumbelli e Bice Mortara Garavelli (a cura di), Parafrasi. Dalla ricerca linguistica alla ricerca psicolinguistica, Alessandria, Edizioni dell'Orso, pp. 169-206.

MARCONI, Diego / BerTinetTo, Pier Marco (1984): «Ma in italiano. Parte prima: Semantica e pragmatica» e «Parte seconda: Proiezioni diacroniche», Lingua e Stile 19, pp. 223-58 e pp. 475-509.

RODRIGUEZ SOMOLINOS, Amalia (2002): «Ainz et mais en ancien français», Romania, 120 (3-4), pp. 505-541. 
TraugOTT, Elizabeth Closs (i.c.s.): «Do semantic modal maps have a role in a constructionalization approach to modals?», in Bert Cappelle, Ilse Depraetere (eds.), Special issue on Modals and Construction Grammar of Constructions and Frames.

TRAUGOTT, Elizabeth Closs / KÖNIG Ekkehard (1991): «The semantics-pragmatics of grammaticalization revisited», in Elizabeth ClossTraugott, Bernd Heine (eds.), Approaches to grammaticalization, vol 1, Amsterdam, John Benjamins, pp. 189-218.

VISCONTI, Jacqueline (2007): «Lessico e contesto: sulla diacronia di mica», in Anna-Maria De Cesare, Angela Ferrari (a cura di), Lessico, grammatica, testualità, Acta Romanica Basiliensia 18, pp. 203-221.

VISCONTI, Jacqueline (2015): «Contesto e co-testo nel mutamento semantico», in Angela Ferrari, Letizia Lala, Roska Stojmenova (a cura di), Testualità. Fondamenti, unità, relazioni, Firenze, Cesati, pp. 263-272.

\section{Corpora}

C-ORAL-ROM = Integrated Reference Corpora for Spoken Romance Languages, a cura di Emanuela Cresti e Massimo Moneglia, Amsterdam/Philadelphia, John Benjamins, 2005.

LIP = Lessico di frequenza dell'italiano parlato, a cura di Tullio De Mauro, Federico Mancini, Massimo Vedovelli e Miriam Voghera, Milano, ETAS Libri, 1993.

LIZ = Letteratura Italiana Zanichelli, version 4.0, a cura di Pasquale Stoppelli e Eugenio Picchi, Bologna, Zanichelli, 2001 (CD-ROM).

OVI = Opera del Vocabolario Italiano-Tesoro della Lingua Italiana delle Origini. Firenze, Consiglio nazionale delle Ricerche / Accademia della Crusca, 1965 $<$ http://www.ovi.cnr.it $>$.

RIDIRE $=$ Risorse Dinamiche dell'Italiano $<$ http://lablita.dit.unifi.it/projects/ RIDIRE $>$. 